\title{
The "Embowire" Technique: Concept and Description of a Novel Embolization Technique for Narrow Vessels
}

\author{
Ruben Lopez-Benitez • Oliver Rusch • \\ Johannes Heverhagen • Kara Levent • \\ Hoppe Hanno · Christa Geiser · Joachim Kettenbach
}

Received: 24 October 2012/ Accepted: 10 February 2013/Published online: 12 April 2013

(C) Springer Science+Business Media New York and the Cardiovascular and Interventional Radiological Society of Europe (CIRSE) 2013

\begin{abstract}
We describe a new, useful embolization technique applied to occlude narrow vessel branches $(\leq 1.5 \mathrm{~mm}$ $\left(0.06^{\prime \prime}\right)$ in diameter) by deployment of one hydrocoil, through a microcatheter, in a way similar to the way in which one might navigate through the vascular lumen with a guidewire.
\end{abstract}

Keywords Embolization · Embolotherapy ·

Radioembolization · Hemorrhage

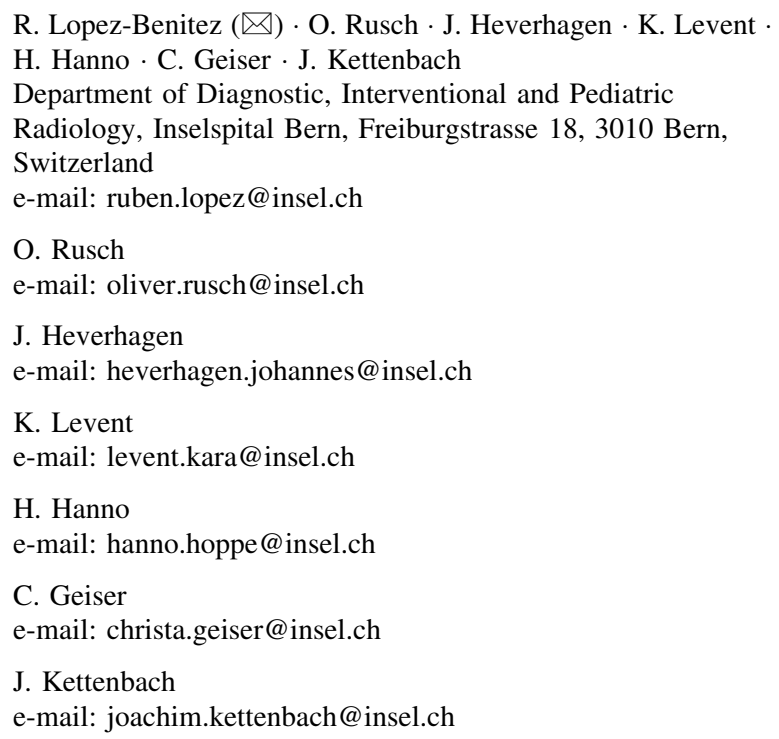

e-mail: joachim.kettenbach@insel.ch

\footnotetext{
We are grateful for support by the Ludwig-Boltzman-Institute of Vienna and to Mary McAllister, John Hopkins University Hospital, Baltimore MD, for her assistance.
}

\section{Introduction}

Endovascular occlusion using microcoils is a well-established treatment for hemorrhagic indications or when endovascular protection is required before tumor embolization with microparticles or radioembolic agents [1].

Bioactive coils are designed to provide a greater occlusion rate of vessels, resulting in a lower rate of rebleeding and retreatment [2]. Hydrocoils (MicroVention Inc., Tustin, CA) are embolic devices that consist of a platinum wire surrounded by a layer of acrylic hydrogel polymer. One advantage of the hydrocoil is its improved coil-packing density due to expansion of the hydrogel polymer after contact with liquid or blood [3].

The hydrogel polymer increases the effective coil diameter up to several times its original diameter (at least three times). Therefore, reliable vessel occlusion depends not so much on the thrombogenicity of the blood and formation of a thrombus, but rather, is achieved by expansion of the hydrogel polymer [4-6]. This particular feature can be helpful in patients who have impaired coagulation when other coil materials would fail or need longer times and more coils to induce a vascular thrombosis [7].

Furthermore, in small and tortuous vessels, it might be difficult to access the vascular bed, despite using microcatheters, even if a guidewire was able to access distant vessel areas; pushing forward to follow the guidewire with a microcatheter may then be difficult through tortuous vessels, due to the soft-tip of the microcatheter and lack of stable pushability.

We describe a new, useful embolization technique applied to occlude narrow vessel branches $(\leq 1.5 \mathrm{~mm}$ $\left(0.06^{\prime \prime}\right)$ in diameter) by deployment of one hydrocoil, through a microcatheter, in a way similar to the way in 
which one might navigate through the vascular lumen with a guidewire.

\section{Materials and Methods}

The "Embowire" technique can only be performed under certain technical conditions. First, temperature plays a very important role in the induction of configurational changes in hydrocoils [7]. Previous experimental work demonstrated that increases of temperature in the hydrocoil filament above $55{ }^{\circ} \mathrm{C}$ quickly induce curling of the coil within seconds ( $\sim 3$ s). Deployment of a hydrocoil without previous exposure to higher temperatures results in a temporary straight configuration of the hydrocoil filament inside the vessel.

The straight configuration of a hydrocoil allows it to be navigated through narrow vascular beds like a guidewire. Furthermore, target vessels that cannot be reached by a microcatheter due to spasm or tortuous anatomy might be accessed by a sufficiently long hydrocoil, and therefore, embolized after hydrogel expansion.

Second, all hydrocoils are designed with a preformed "first loop" at the tip of the filament; this first loop facilitates, in normal conditions, the initial binding of the hydrocoil. To perform the Embowire technique, it is mandatory to ensure a guidewire-like filament shape with either a straight or a slight curvature of the tip. Therefore, it is necessary to cut this "first loop," i.e., with a pair of sterile scissors (Figs. 1A-C). The cut should be transversal to the direction of the filament, avoiding the creation of a sharp tip at the distal part of the hydrocoil.

The "first loop" of all hydrocoils has no hydrogel attached to its surface and consists only of platin; thus, there is no risk of damage to the hydrogel cover or damage to the electric deployment mechanism if cut off.
Third, once the hydrocoil filament has been prepared for the deployment, it is only necessary to insert it into the microcatheter and to advance it like a guidewire until the filament reaches the desired area to be occluded.

Fourth, this technique applies to the use of detachable hydrocoils, where it is possible to retrieve and reposition the hydrocoil if advanced beyond the target area.

Since developing this technique, we have been using it successfully in many clinical situations including:

- Retrograde embolization of the right gastric artery for protective occlusion to avoid nontarget embolization in selective-internal-radiation-therapy (SIRT) procedures.

- Treatment of gastrointestinal bleeding from duodenal and gastric vessels.

- Occlusion of small unreachable vessels in vascular arteriovenous-pulmonary fistulas.

- Embolization in acute posttraumatic bleeding of the distal segments of the lumbar arteries and the sacral media artery.

- Embolization in acute retroperitoneal bleeding (usually from lumbar arteries) in patients with an impaired coagulation profile.

We describe three clinical situations in which the "Embowire" technique was successfully used. According to the local Institutional review Board (IRB) advice guidelines, signed agreement for the use of the anonymized clinical information for this manuscript was obtained in every one of these three presented cases.

\section{Case 1}

A 51-year-old male patient with an inoperable 9-cm hepatocellular carcinoma on the left liver lobe was

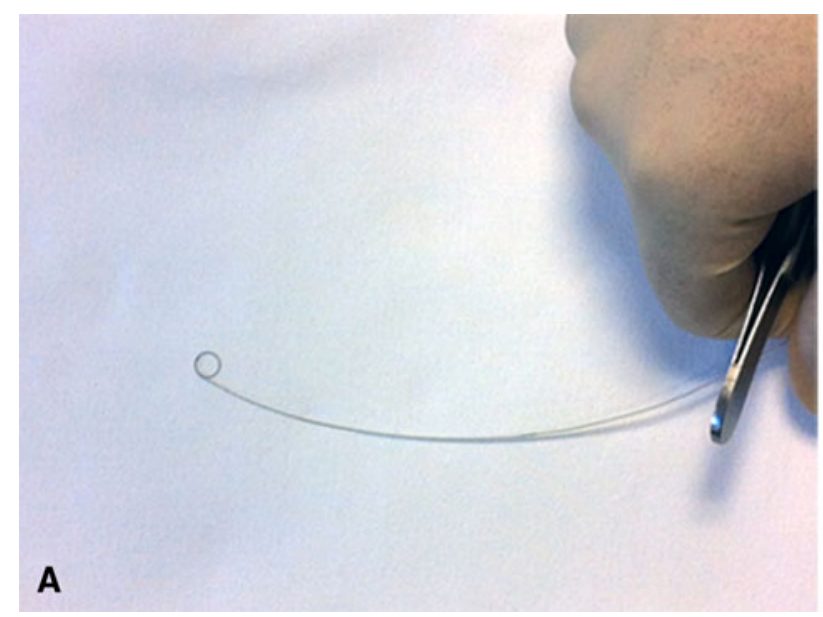

Fig. 1 Preparation of the Hydrocoil for Embowire technique. A All Hydrocoils (MicroVention Inc., Tustin, CA) are provided with a preformed loop at the tip of the filament. B By using sterile scissors,

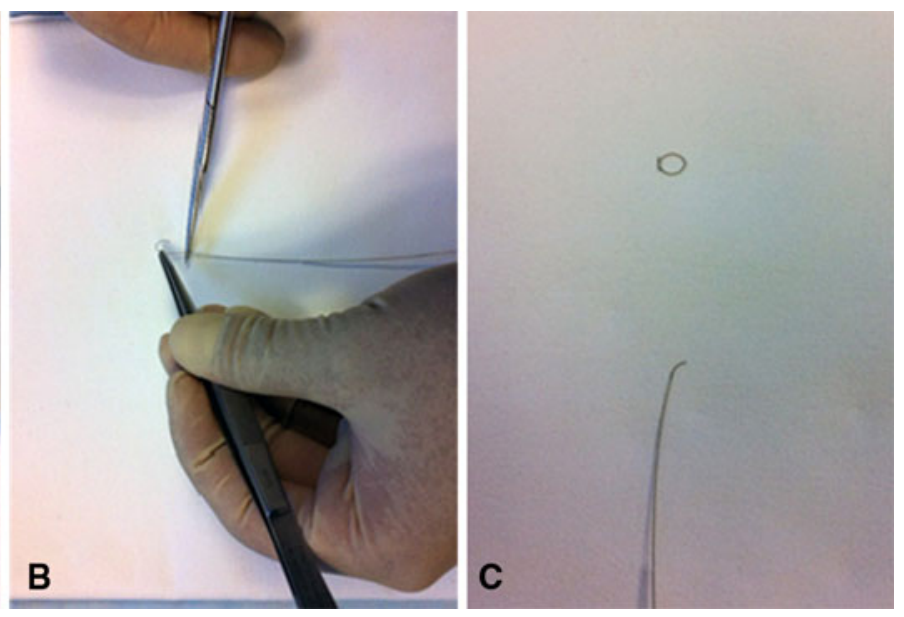

the first loop must be cut off. C Finally, the Hydrocoil acquires a very similar configuration as a guidewire 


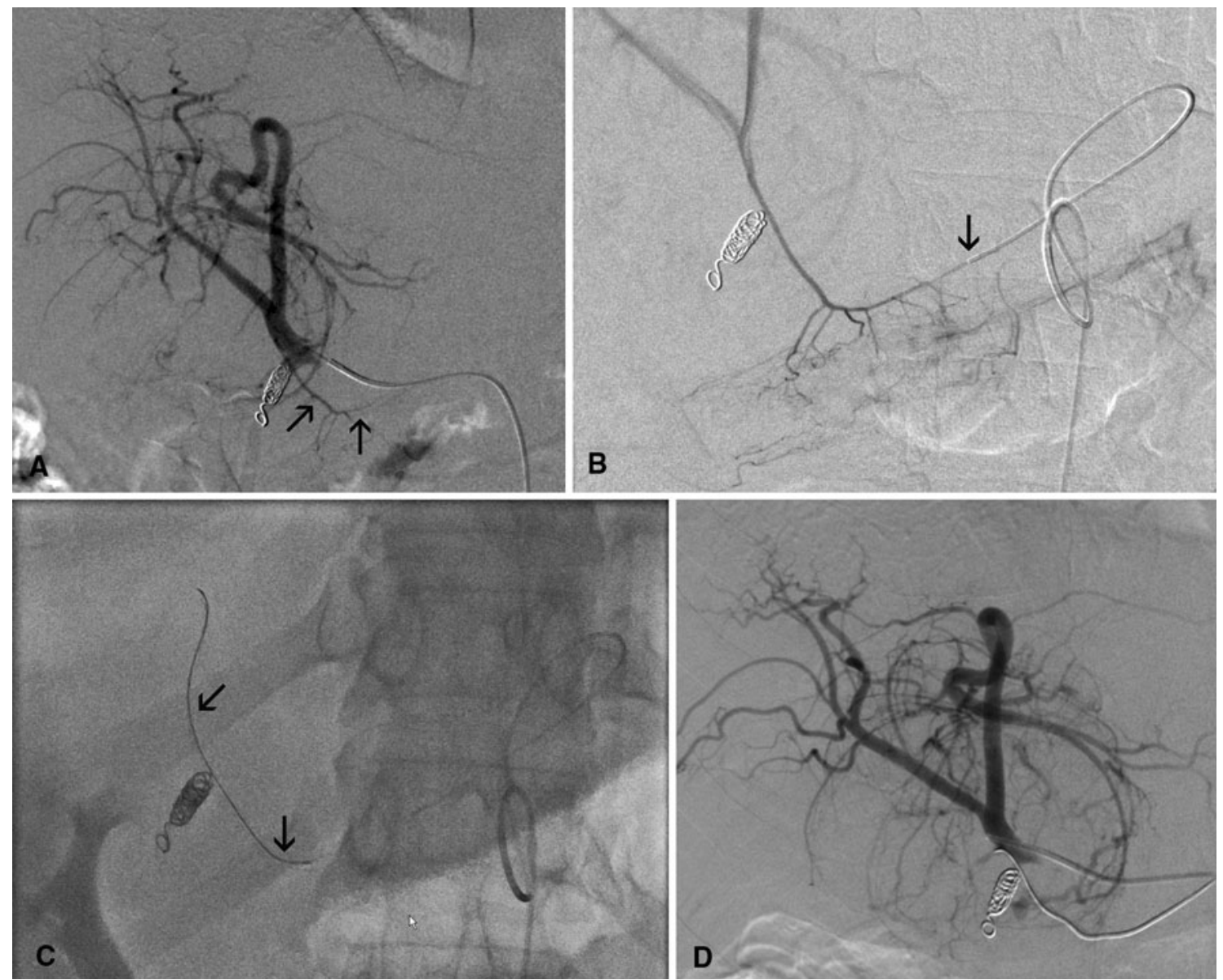

Fig. 2 A Selective digital substraction angiography (DSA) through the proper hepatic artery shows an open right gastric artery (RGA) (black arrows) where antegrade access using a microcatheter was not possible. B From the left gastric artery (LGA), the microcatheter tip (black arrow) could not be advanced further in retrograde fashion to the origin of the RGA. C Using the Embowire technique, the hydrocoil filament (black arrows) was advanced in retrograde fashion through the RGA and even further, deep into the left hepatic artery. D Control DSA shows a complete occlusion of the RGA, without side branches arising from this vessel admitted to our service for a radioembolization procedure (SIRT). During the SIRT workup, the right gastric artery (RGA) was identified, unfortunately, only a few millimeters away from the origin of the left hepatic artery where the radioactive spheres were supposed to be injected (Fig. 2A).

To prevent particle reflux into the RGA, it was decided to occlude this vessel in an antegrade fashion from its origin in the main hepatic artery. Unfortunately, due to a very closed angulation of the RGA origin, it was not possible to provide stable access to the target vessel. We decided to perform a retrograde catheterization of this vessel through the left gastric artery (LGA) using a 0.8-mm (2.4 F) microcatheter (Progreat; Terumo-Europe. NV).

Despite a relatively easy catheterization of the marginal artery in the small stomach curvature, it was not possible to advance the microcatheter any further through the last
$7 \mathrm{~cm}$ of the RGA to its origin; therefore, the risk of nontarget embolization remained (Fig. 2B).

A $6 \times 20-\mathrm{mm}$ detachable Hydrocoil ${ }^{\mathrm{TM}}$ (MicroVention Inc., Tustin, CA) was prepared for the "Embowire" technique by cutting the distal tip of it, and it was possible to advance the hydrocoil all the way through the $7 \mathrm{~cm}$ of the RGA, even up to the left hepatic artery (Fig. 2C).

Once positioned on the embolization target segment, the hydrocoil was detached. After only $1 \mathrm{~min}$, the subsequent control angiography showed no more perfusion in the RGA or within the branches supplied by this vessel. Angiography from the celiac trunk confirmed a complete occlusion of the RGA within 3 min after deployment (Fig. 2D). The Tc-99 scintigraphy (as part of the SIRT pretreatment planning), performed after the RGA coiling, did not show any gastric up-take.

On the late angiography, after 7 days and just before starting the SIRT, the RGA remained occluded and the therapy was performed with no complications. 

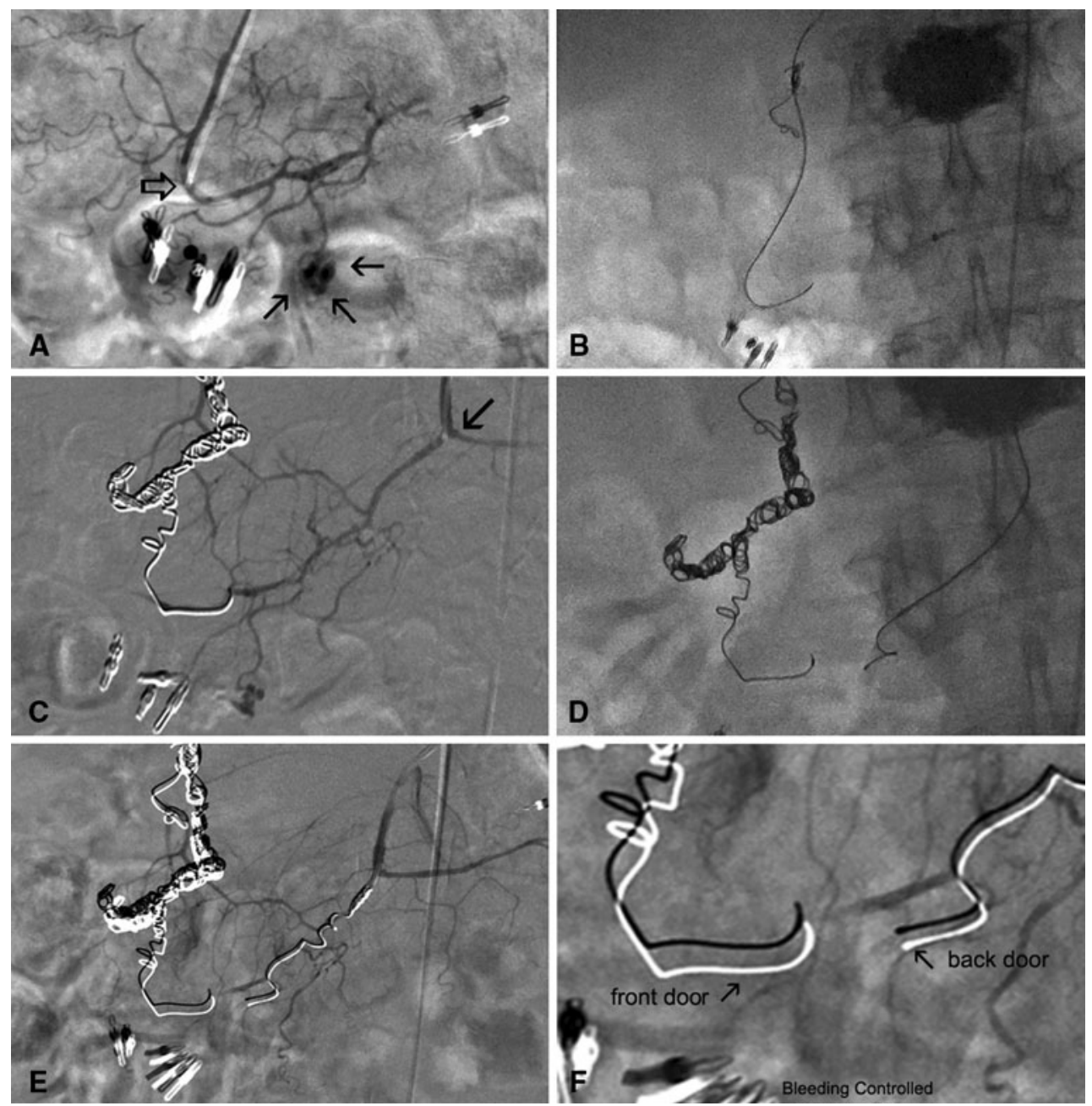

Fig. 3 A Duodenal vessel bleeding branch originate from the gastro duodenal artery (GDA); the tip of the $0.8-\mathrm{mm}$ microcatheter (open arrow) could not be advanced exactly to the bleeding site (black arrows). B "Embowire" technique embolization performed with a $0.018^{\prime \prime} \times 3-\mathrm{cm}$ length hydrocoil from the GDA-branch. C Catheterization of a duodenal branch from the superior mesenteric artery (SMA) showing the duodenal bleeding. As in image $3 \mathrm{~A}$, the

\section{Case 2}

A 69-year-old male patient presented with refractory duodenal bleeding, which originated from the tiny branches of the GDA and the superior mesenteric artery (SMA; Fig. 3A). Despite proximal catheterization of the main bleeding branch originating from the GDA, the tip of the 0.8-mm (2.4 F) microcatheter could not be advanced exactly to the bleeding point.

Initially, the "Embowire" embolization technique was performed using a hydrocoil (of an approximately length of $3 \mathrm{~cm}$ with the use of a $4-\mathrm{mm} \times 10-\mathrm{cm}$ coil diameter), finally reaching the bleeding point supplied by a branch of the GDA. Although with this technique it was possible to microcatheter could not be advanced selectively to the bleeding vessel (black arrow). D-F The "Embowire" technique shows the second hydrocoil filament successfully advanced to the bleeding site (front door and back door occluded); the bleeding was successfully stopped (*some images are blurred due to involuntary respiratory movement in a severely impaired patient)

occlude the "front door" of the bleeding source (from the GDA), the supply from the SMA branches remained patent (Figs. 3B, C).

Therefore, in a second step, a catheterization of the duodenal branch (back-door concept) originating from the SMA was planned. Unfortunately, the same situation occurred again and the microcatheter could not be advanced to reach the bleeding site. The "Embowire" technique was then performed and the hydrocoil filament could be advanced and placed successfully at the bleeding point: thus, the "back door" of the bleeding vessel was closed (Figs. 3D-F).

In the control angiography, no active bleeding was identified after the embolization. The patient soon achieved 
a hemodynamically stable status and no further signs or symptoms of bleeding were identified.

\section{Case 3}

A 4-month-old female patient with a complex congenital arteriopulmonary malformation was referred to our service for embolization. Multiple feeding arteries between the liver, the intercostal, and the lumbar arteries supplied a pulmonary malformation with a high-flow fistula component (Fig. 4A).

In this child, the lumbar arteries were very narrow, which did not allow for the passage of an $0.8-\mathrm{mm}$ microcatheter (Progreat; Terumo-Europe. NV) into the feeding vessel of the arteriopulmonary fistula (Fig. 4B).

The "Embowire" technique was then applied from the point where the microcatheter could not be advanced any further. Then, a short, detachable hydrocoil $(4 \mathrm{~mm} \times 10 \mathrm{~cm})$ was advanced through the complete vessel-bed and positioned exactly at the point of entrance of the arteriopulmonary fistula (Figs. 4C, D). After less
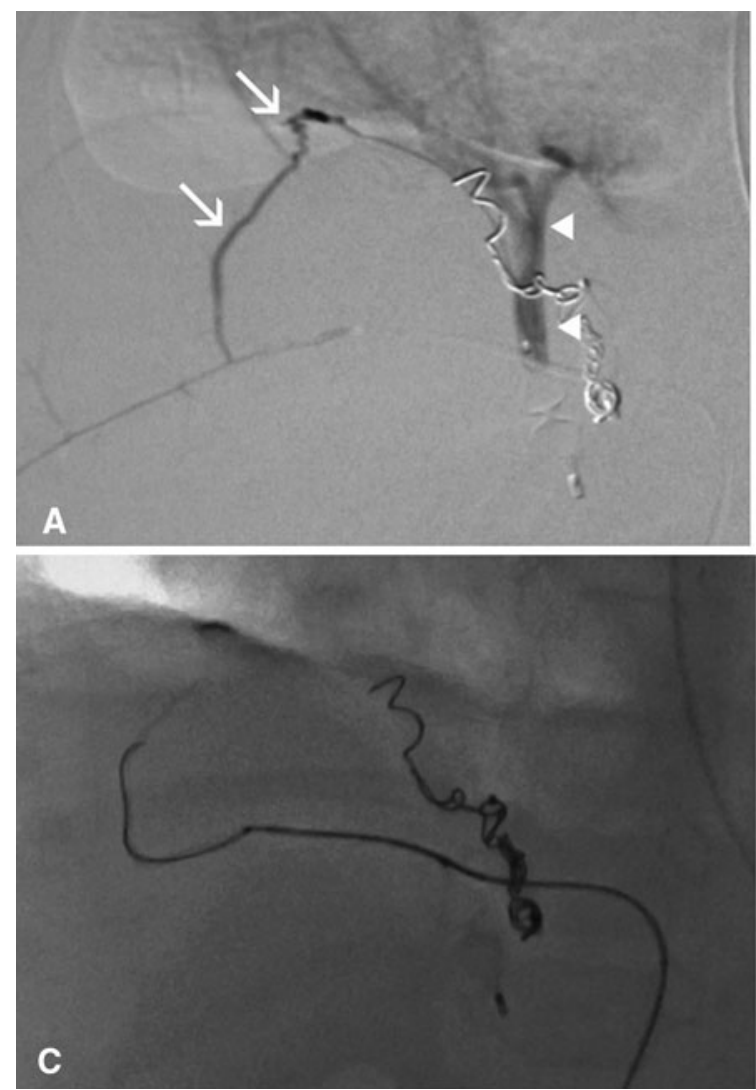

Fig. 4 Four-month-old female with congenital arteriopulmonary malformation. A Feeding intercostal artery supplying communication vessel (white arrows) to an arteriopulmonary malformation vessel (white arrowheads). B. Tip of the microcatheter advanced only $2 \mathrm{~cm}$ proximal to the supplier vessel (white arrow). C Embowire technique than $5 \mathrm{~min}$, the expansion of the hydrogel led to a complete obliteration of the embolized vascular segment and no more filling of the pulmonary vessel. Despite the good interventional results, the patient died 2 weeks later of respiratory failure secondary to pneumonic complications.

\section{Results}

We successfully performed the vascular occlusion of the above-mentioned cases with the use of the Embowire technique. This method was always chosen when the vascular target (i.e., bleeding source) could not be reached with the microcatheter, making a durable embolization with only a proximal vascular occlusion unsafe.

In all of these cases, angiographic evidence of vascular occlusion was present, as well as complete exclusion of extravascular contrast medium, after less than $5 \mathrm{~min}$. None of these cases showed any clinical signs of distal organ ischemia or late migration of the implanted material. There also were no allergic reactions or postembolization syndrome symptoms.

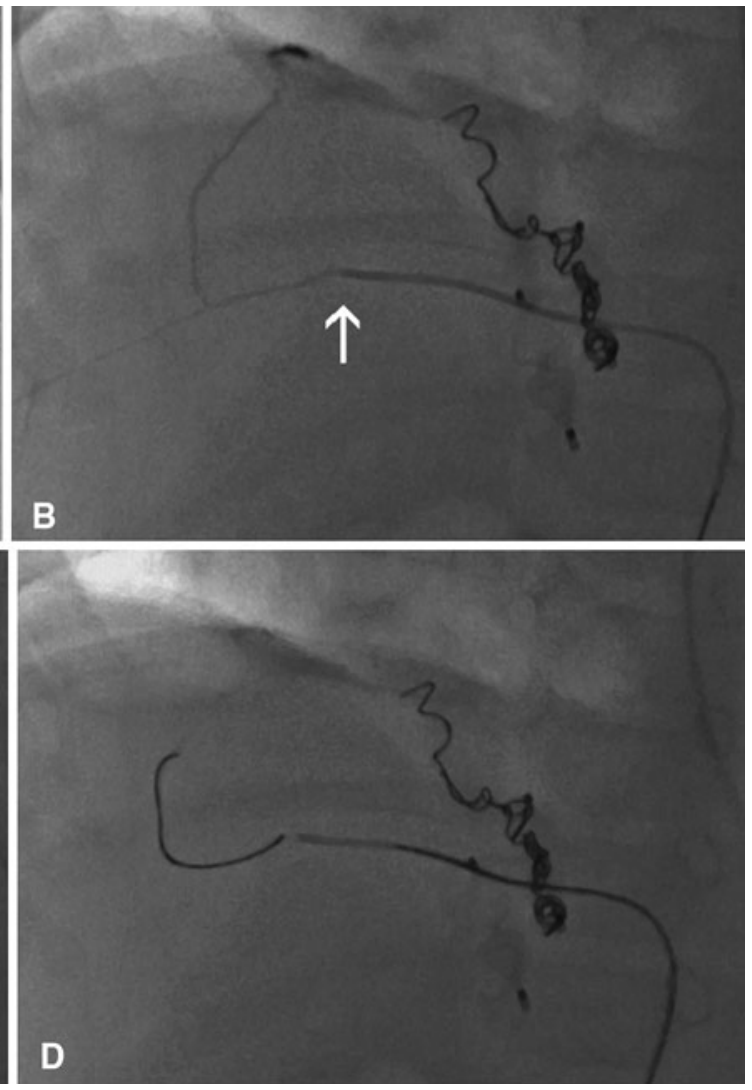

showed at the moment when a $0.018^{\prime \prime} \times 3-\mathrm{cm}$ length hydrocoil filament attached to the deployment wire. D. Detached hydrocoil filament finally positioned into the malformation supplying vessel; the hydrogel expansion was sufficient to occlude the communication vessel into the pulmonary malformation 


\section{Discussion}

The above-described technique is known in our interventional group as the "Embowire" technique. The name derives from the conjunction of two words: "Embo" because of the embolization capabilities of the hydrocoil (embolization through expansion of the hydrogel), and "wire" referring to the manner of implantation of the hydrocoil filament, similar to a guidewire.

The main principle of this technique is the vascular occlusion of narrow vessels induced by the expansion of the hydrogel coating. Video analysis of the expansion of the hydrogel coating on a 0.018 " platin filament shows a final expanded diameter of at least three times the original diameter. Thus, we recommend the "Embowire" technique to be applied only in those vessels with a maximal diameter of $1.5 \mathrm{~mm}$ ( $\leq 0.06 \mathrm{inch}$ ). Attempts to perform the "Embowire" technique in vessels with wider diameters could result in an incomplete vascular occlusion.

Although there are 0.035-inch hydrocoils on the market, we used only the $0.018^{\prime \prime}$ detachable hydrocoils to perform this technique, because we determined that this technique is especially useful in distal narrow vessels. In our experience, the "Embowire" technique is particularly useful for reaching vascular segments distal to the tip of a microcatheter, when it is not possible to advance the catheter to the desired vascular target.

Nevertheless, the "Embowire" technique could be limited by circumstances, such as complete vascular spasm or very tortuous vascular anatomy, because in these cases steering the hydrocoil filament as a guidewire may not be possible. Vascular diameters wider than the total expanded diameter of the hydrocoil will result in an incomplete luminal occlusion.

In general, vascular embolization is usually performed by packing the coil material in the vascular target or proximal to the bleeding source if the vessel is unable to be catheterized [8]. However, the use of hydrogel-coated platin coils for the embolization as straight filaments has been only experimentally described [9].

Because this technique is described only with the use of detachable hydrocoils, the risk of migration of the embolization filament is practically nonexistent. Embolic migration of hydrogel casts due to cutting of the first loop of the hydrocoil also is very unlikely because, as already mentioned, the loop consists of only platin, and no hydrogel has been added to the surface in this section of the hydrocoil.
The "Embowire" technique is a very useful technique for reaching narrow vascular segments that have to be embolized, especially when a microcatheter cannot be advanced to the target point. This technique is safe and reproducible; however, skillful training and experience will be necessary to achieve acceptable results.

Acknowledgments The authors are grateful for support by the Ludwig-Boltzman-Institute of Vienna and to Mary McAllister, John Hopkins University Hospital, Baltimore, MD, for her assistance.

Conflict of Interest Lopez-Benitez Ruben, Rusch Oliver, Heverhagen Johannes, Kara Levent, Hoppe Hanno, Geiser Christa, and Kettenbach Joachim have no conflict of interest to disclose.

\section{References}

1. Dudeck O, Bulla K, Wieners G et al (2011) Embolization of the gastroduodenal artery before selective internal radiotherapy: a prospectively randomized trial comparing standard pushable coils with fibered interlock detachable coils. Cardiovasc Intervent Radiol 34(1):74-80

2. Geyik S, Ertugrul O, Yavuz K, Geyik P, Saatci I, Cekirge HS (2010) Comparison of bioactive coils and bare platinum coils for treatment of intracranial aneurysms: a matched-pair analysis. J Neurosurg 112(4):709-713

3. Guimaraes M, Uflacker R, Garretson JS et al (2011) Angiographic and histologic comparison of injectable, expansile hydrogel embolic and pushable AZUR embolic devices in porcine arteries. J Vasc Interv Radiol 22(11):1619-1624 e1611

4. Link DP, Mourtada FA, Jackson J, Blashka K, Samphilipo MA (1994) Hydrogel embolic agents. Theory and practice of adding radio-opacity. Invest Radiol 29(8):746-751

5. Link DP, Strandberg JD, Virmani R, Blashka K, Mourtada F, Samphilipo MA (1996) Histopathologic appearance of arterial occlusions with hydrogel and polyvinyl alcohol embolic material in domestic swine. J Vasc Interv Radiol 7(6):897-905

6. Killer M, Arthur AS, Barr JD, Richling B, Cruise GM (2010) Histomorphology of thrombus organization, neointima formation, and foreign body response in retrieved human aneurysms treated with hydrocoil devices. J Biomed Mater Res B 94(2):486-492

7. Lopez-Benitez R, Hallscheidt P, Kratochwil C et al (2012) Protective embolization of the gastroduodenal artery with a oneHydroCoil technique in radioembolization procedures. Cardiovasc Intervent Radiol 36(1):105-110

8. White PM, Lewis SC, Nahser H, Sellar RJ, Goddard T, Gholkar A (2008) HydroCoil Endovascular Aneurysm Occlusion and Packing Study (HELPS trial): procedural safety and operator-assessed efficacy results. AJNR Am J Neuroradiol 29(2):217-223

9. Killer M, Keeley EM, Cruise GM, Schmitt A, McCoy MR (2011) MR imaging of hydrogel filament embolic devices loaded with superparamagnetic iron oxide or gadolinium. Neuroradiology 53(6):449-456 